ORIENTAL JOURNAL OF CHEMISTRY

An International Open Free Access, Peer Reviewed Research Journal

www.orientjchem.org
ISSN: 0970-020 X

CODEN: OJCHEG

2013, Vol. 29, No. (4):

Pg. 1475-1487

\title{
Synthesis of Flavone Skeleton by Different Methods
}

\author{
R.B. KSHATRIYA, Y.I. SHAIKH and G.M. NAZERUDDIN* \\ Department of Chemistry (P.G. \& Research Centre), Poona College ofArts, \\ Science \& Commerce, Pune, India. \\ Corresponding author E-mail: rbkshatriya123@gmail.com \\ http://dx.doi.org/10.13005/ojc/290425
}

(Received: October 01, 2013; Accepted: November 13, 2013)

\begin{abstract}
Flavones (flavus = yellow), are a class of flavonoids based on the backbone of 2-phenylchromen-4-one. Flavones are mainly found in cereals and herbs. Flavones are biologically active compounds. Therefore number of synthetic methods were developed. In this mini revive we have tried to cover various synthetic strategies for the synthesis of flavones. Some of the well known methods used for synthesis of flavones are Baker \& Venkatraman synthesis and Claisen-Schmidt condensation.
\end{abstract}

Key words: Flavones, Biologically Active Compounds, Synthetic Methods

\section{INTRODUCTION}

Flavones (flavus $=$ yellow), are a class of flavonoids based on the backbone of 2-phenylchromen-4-one Apart from flavones other flavonoids are isoflavonoids, derived from 3-phenylchromen-4-one structure neoflavonoids, derived from 4-phenylcoumarine structure. The three flavonoid classes are all ketone-containing compounds, and as such, are anthoxanthins (flavones and flavonols)

Flavones are well known for their various biological activities such as anticancer1 Anti<smiles>O=c1cc(-c2ccccc2)oc2ccccc12</smiles>

Flavone<smiles>O=c1c(-c2ccccc2)coc2ccccc12</smiles>

Isoflavone<smiles>O=c1cc(-c2ccccc2)c2ccccc2o1</smiles>

Neoflavone 


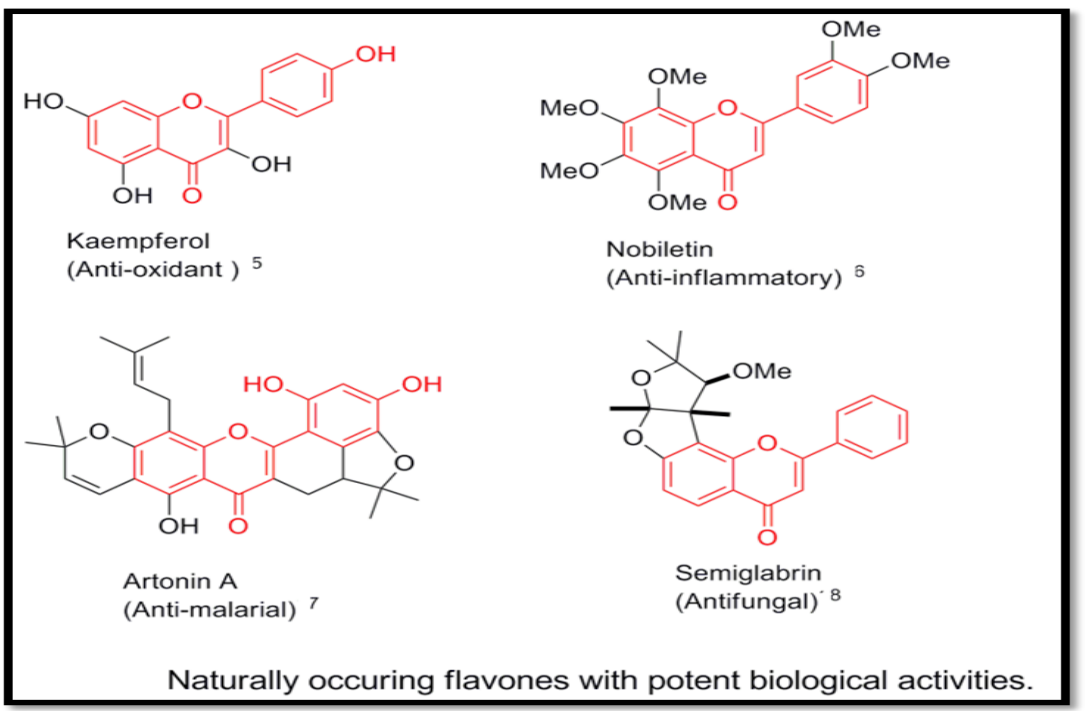

inflammatory2, anti-osteoporotic3, anti-diabetic4, etc. some of the examples as shown as under.

\section{Synthetic strategies of flaovones}

Traditionally, flavones have been prepared by BakerVenkatramanrearrangement and Claisen-Schmidt condensation.which involves the conversion of 2 hydroxyacetophenones into benzoyl esters, followed by rearrangement in base to1,3diphenylpropane1,3diones which upon cyclization under acidic conditions furnishes flavones. On the other hand hydroxychalcone synthesized from 2 hydroxyacetophenone anbenzaldehyde under ClaisenSchmidt conditions can undergo oxidative cyclization to furnish flavones ring.

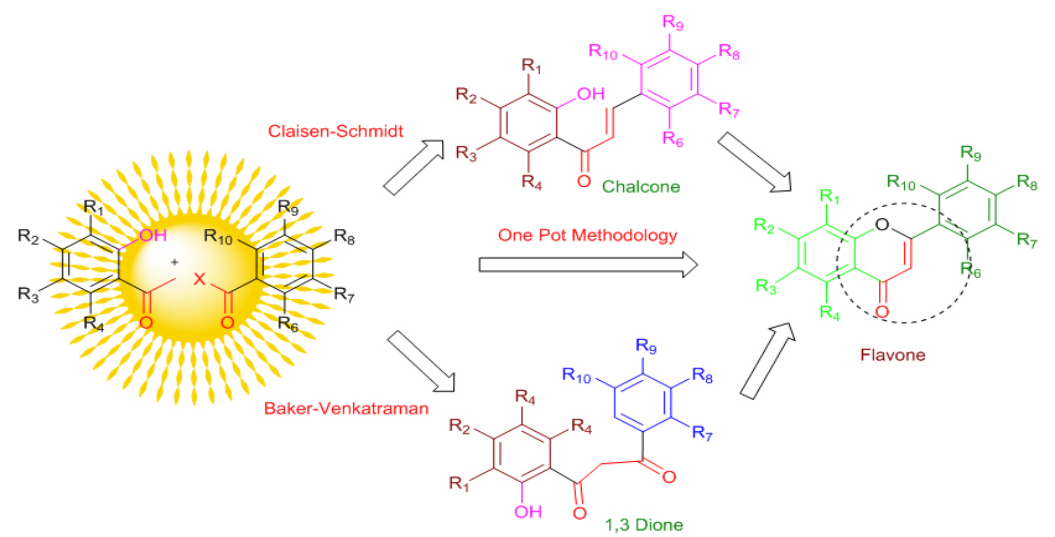

Fig. 1: Basic reactions for the synthesis of flavones

Basic schemes related to synthesis of flavones is mentioned below (Scheme 1-43),<smiles></smiles><smiles>[R]C#C</smiles>

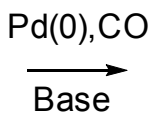<smiles>[R2]c1cc(=O)c2ccccc2o1</smiles>

Scheme 1: Palladium catalysed synthesis is carried out in sence of basic environment by Hua \& Yang ${ }^{9}$ 
<smiles>[R]c1cc(-c2cc(=O)c3c(O)cc(O)cc3o2)c([R])c([R])c1[R]</smiles>

Scheme 2: Solvent free synthesiso f flavone is carrie out by Julia \& co-workers ${ }^{10}$

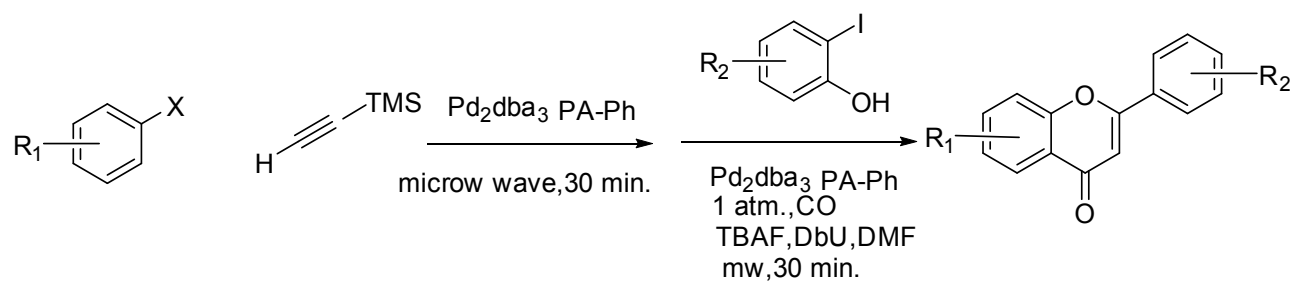

Scheme 3: Flavones via a Micro-Assisted, One-Pot Sonogashira"Carbonylation" Annulation Reactionis used by E.Awuah \& A.Capretta ${ }^{11}$<smiles>[R]c1ccc(C(=O)C([X])(Cl)C(=O)c2cc([R4])c([R3])c([R])c2)cc1</smiles>

Scheme 4: Photo cyclization of 2-Chloro-Substituted 1,3-Diarylpropan-1,3-diones to Flavones is invented by B.Kosmrrji \& co-workers ${ }^{12}$<smiles>[R]c1ccc(O)c(C(=O)CC(=O)c2ccccc2)c1</smiles>

Scheme 5: Coversion of intermediate 1,3 dione is carried by G.Romanelli \& co-workers ${ }^{13}$<smiles>O=c1ccoc2ccccc12</smiles>

1. TMP2Zn.2MgCl2.2LiCl

THF, $-30{ }^{\circ} \mathrm{C}$

2. $\mathrm{E}^{+}$<smiles>O=c1cc(F)oc2ccccc12</smiles>

$\mathrm{E}=$ allyl, acyl, aryl

Scheme 6: Alkene hydrogen is replaced by L.Klier \& T.Bresser ${ }^{14}$ 
<smiles>[R]C(=O)Oc1ccccc1C(=O)O[SnH3]</smiles>

Scheme 7: A Novel Synthesis of $4 \mathrm{H}$-Chromen-4-ones via Intramolecular Wittig Reaction is used for the synthesis of flavones ${ }^{15}$

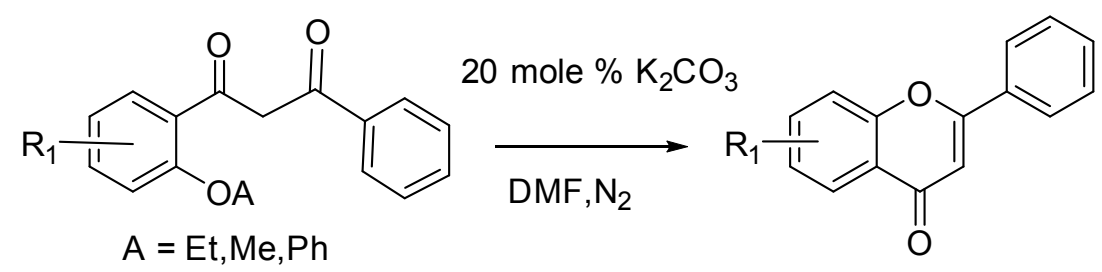

Scheme 8: This invention converts 1,3 dione into flavones. Only base is used for this purpose ${ }^{16}$

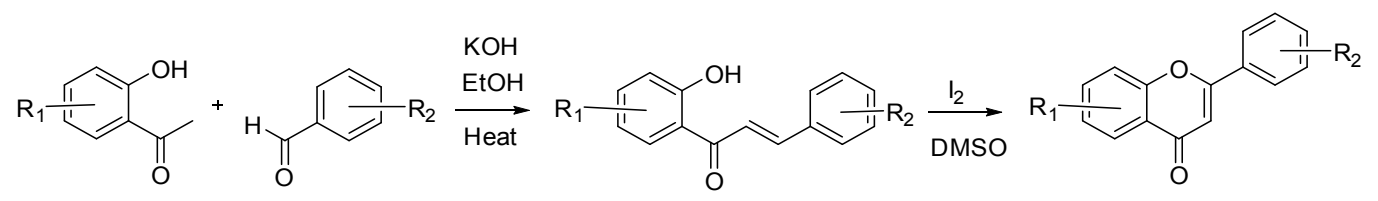

Scheme 9: Koneni \& his group first time invented flavones in which oxygen of flavone come from watr molecule ${ }^{17}$<smiles>[R]c1cccc(-c2cc(=O)c3ccc([R])cc3oc2=O)c1</smiles>

Scheme10: A two step synthesis of flavones via Wacker oxidation is carried out in this process ${ }^{18}$<smiles>[R][R]1ccc(O)c(C(=O)CC([R])=O)c1</smiles>

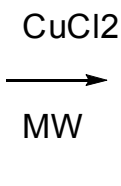<smiles></smiles>

Scheme 11: G.Kabalka \& A.Meredy carried microwave assisted synthesis of flavones.Copper chloride is used as a catalyst for this process ${ }^{19}$ 
<smiles>[R]C(=O)CC(=O)c1cc[R1]([H])cc1O</smiles>

Scheme 12: Photo-Wittig reaction is apllied for the synthesis of flavones ${ }^{20}$<smiles>[R]c1ccc(-c2cc(=O)c3cc([R])c([R2])cc3o2)cc1</smiles>

Scheme13.0xidative cyclisation of chalcone to flavone is carried out for the synthesis of flavones. Here $\mathrm{n}$-tetrabutylammonium tribromide is used as a catalys $\mathbf{t}^{21}$<smiles>[R]c1cc(O)c(C(=O)/C=C/c2cc([R4])c([R3])c([R])c2[R])cc1[R6]</smiles>

$\underset{\text { 2. }}{\stackrel{\text { Et } 3 \mathrm{~N}, \mathrm{~K} 2 \mathrm{CO} 3}{\longrightarrow}}$

3. $0.1 \mathrm{M}, \mathrm{NaOMe}, \mathrm{NaOH}$<smiles>[R]c1cc2oc(-c3cc([R4])c([R3])c([R])c3[R])cc(=O)c2cc1[R6]</smiles>

Scheme 14: 2'allyoxy chalcone undergoes oxidative coupling when treated with iodine \& DMSO$^{22}$<smiles>[R2]c1ccc(/C=C/C(=O)c2cc([R3])c([R3])cc2OCC=C)cc1[R19]</smiles><smiles>[R4]c1ccc(-c2cc(=O)c3cc([R])c([R3])cc3o2)cc1[R]</smiles>

Scheme 15: Palladiumacetate is used catalyst for the synthesis of flavones ${ }^{23}$<smiles>O=c1ccoc2ccccc12</smiles>
$\mathrm{Ar}-\mathrm{H}, \mathrm{Pd}(\mathrm{OAc})_{2}, \mathrm{AgOAC}$ $\mathrm{PivOH}, \mathrm{CsOPiv}, 110^{\circ} \mathrm{C}$<smiles>O=c1cc(-c2ccccc2)oc2ccccc12</smiles>
annulation of 2 bromo phenols \&terminal alkynes is carried out ${ }^{24}$ 


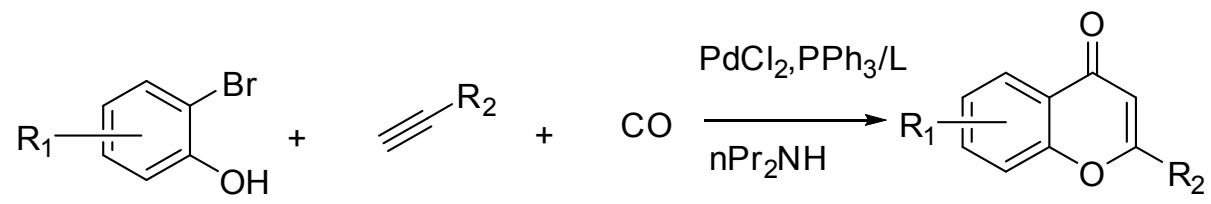

Scheme 17. Ganguly's synthesis includes synthesis of flavones using

O-hydroxy acetophenone \& acetyl chloride as a precursor ${ }^{25}$<smiles>[R]c1cc([R2])c(C(C)=O)c(O)c1</smiles><smiles>[R]C(=O)Cl</smiles><smiles>[R3]C(=O)c1c([R])oc2cc([R])cc([R2])c2c1=O</smiles><smiles>[R3]c1cc(=O)c2cc([R2])c([R])cc2o1</smiles>

Scheme18: One pot synthesis of flavones using ferric chloride is efficient method carrid out by Rajiv Karmarkar \& co-worker ${ }^{26}$<smiles></smiles>

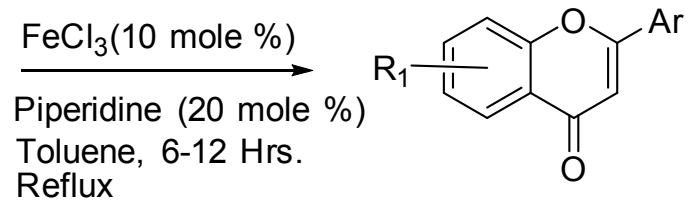

Scheme19: Silica supported lewis acids indium chloride \& indium bromide undergoes oxidative coupling to give flavones ${ }^{27}$<smiles>[R]c1ccc(-c2cc(=O)c3cc(Br)ccc3oc2=O)cc1</smiles>

Scheme 20: Wet acetone is efficient catalyst for the one pot synthesis of flavones from 2-hydroxy acetophenone $\&$ acetyl chloride ${ }^{28}$<smiles>[R]O[R4](=O)C(C)=O</smiles><smiles>[R]c1ccc(-c2cc(=O)c3c([R])cc([R])cc3o2)cc1</smiles>

Major<smiles>[R]c1ccc(C(=O)c2c(-c3ccc([R])cc3)oc3cc([R])c([R])c([R])c3c2=O)cc1</smiles>

Scheme 21: Formation of 1,3 dione using LiHDMs followed by cyclisation using acid catalyst is achived ${ }^{29}$ 


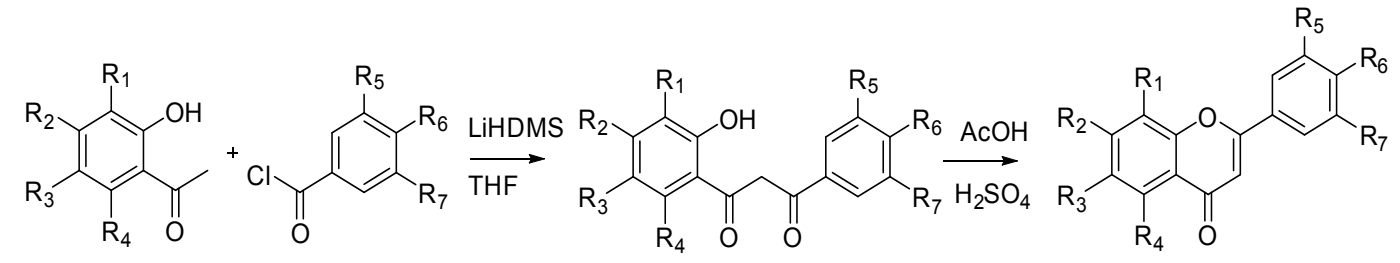

Scheme 22: Carbonylative couplig using Pd catalyst is invention of this method ${ }^{30}$

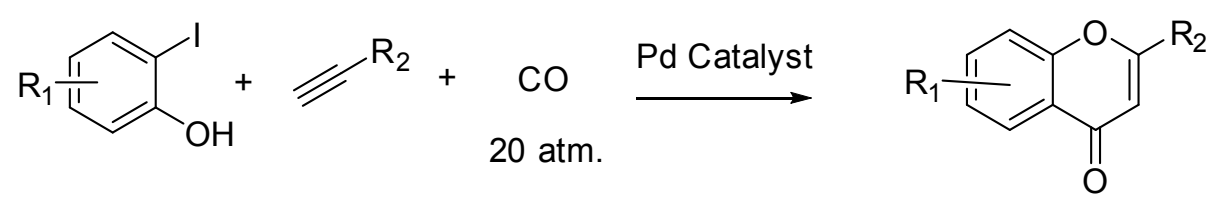

Scheme 23: Daniel etal31suggested the following methodology consisting of five steps<smiles>C=CCc1cccc(C=O)c1O</smiles><smiles>O=[N+]([O-])C(Cl)=Cc1ccccc1</smiles><smiles>Nc1c(-c2ccccc2)oc2c(CC(=O)O)cccc2c1=O</smiles>

Scheme 24: lodo \& bromo derivatives of flavones were synthesized by this method ${ }^{32}$

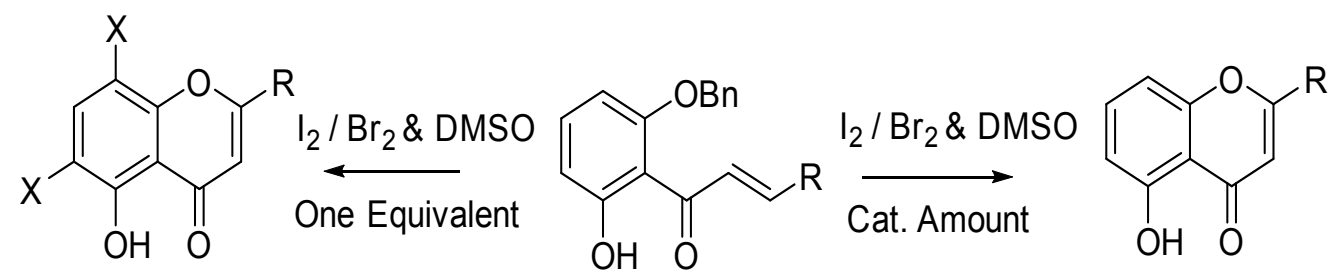

Scheme 25: Oxidative cyclisation followed by bromination is carried out by this process ${ }^{33}$<smiles>[R]c1cc([R2])c(C(=O)/C=C/c2cc([R6])c([R5])c([R3])c2[R3])c(O)c1</smiles>

1. $\mathrm{V}_{2} \mathrm{O}_{5}, \mathrm{H}_{2} \mathrm{O}_{2} / \mathrm{NH}_{4} \mathrm{Br}$

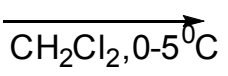
2.0.2 $\mathrm{M} \mathrm{KOH}, \mathrm{EtOH} . \mathrm{H}_{2} \mathrm{O}(4: 1)$<smiles>[R5]c1cc(-c2cc(=O)c3c([R])c([Y])c([R])c([X])c3o2)c([R3])c([R4])c1[R5]</smiles>

Scheme 26: Base is used for cyclisation of inermediate to flavone ${ }^{34}$ 


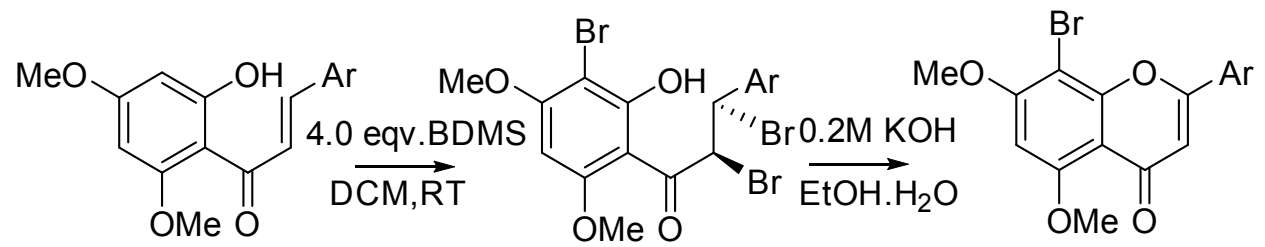

Scheme 27: Wittig reaction is applied for the synthesis of flavones ${ }^{35}$<smiles>[R]c1cc(C(=O)C=[PbH])c(O)c([R1])c1[R]</smiles>

1. RCOCI,Pyridine

2. $\mathrm{MeONa} / \mathrm{MeOH}$<smiles></smiles>

Scheme 28: Frédéric et a ${ }^{36}$ suggested<smiles>[R]C(=O)C(=O)c1c([R])cc([R])cc1O</smiles>

Scheme 29: Dhanapalan $\mathrm{N}^{37}$ et al and Scheme ${ }^{30}$. Yoshida et $\mathbf{a l}^{38}$ suggested the following methodologies respectively<smiles>[R4]c1cc(-c2cc(=O)c3ccccc3o2)c([R])c([R])c1C(=O)O</smiles>

Scheme 30:<smiles>[R]C#CC(=O)c1c([R4])c([R3])c([R])c([R1])c1O</smiles><smiles>[R]c1cc(=O)c2c([R4])c([R3])c([R])c([R])c2o1</smiles>

Scheme 31: Hydrogen peroxide is used as catalyst for this one pot method ${ }^{39}$ 
<smiles>COC(=O)C(C)COC(=O)c1ccc(OC)cc1O</smiles>

Scheme 32: Lewis acid ferric chloride is capplied for the synthesis of flavones via oxidative coupling by Kumar \& Perumal ${ }^{40}$

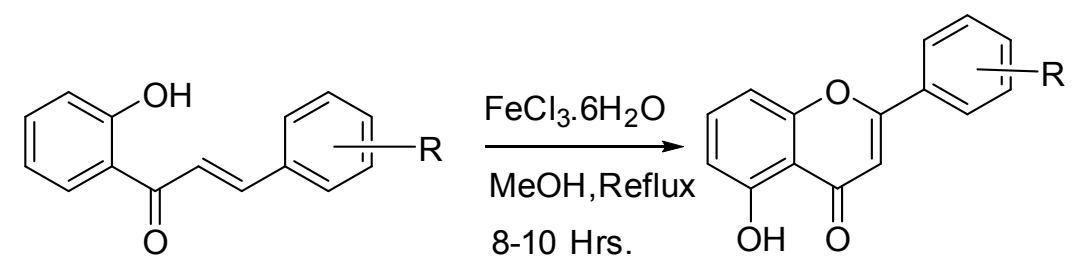

Scheme 33: Zanwar,M. R41suggested the following methodology<smiles>[R7]c1ccc(/C=C/[N+](=O)[O-])cc1</smiles><smiles>[R20]C(=O)c1c(-c2ccc([R])cc2)oc2cc[R1]cc2c1=O</smiles>

Scheme 34: Yitterbium triflate is ude for the one pot synthesi of flavones in this paper ${ }^{42}$<smiles>[X]c1ccc(O)cc1</smiles>

Scheme 35: Suzuki-Miyaura coupling used for the synthesis of flavone by Kraus \& Gupta ${ }^{43}$

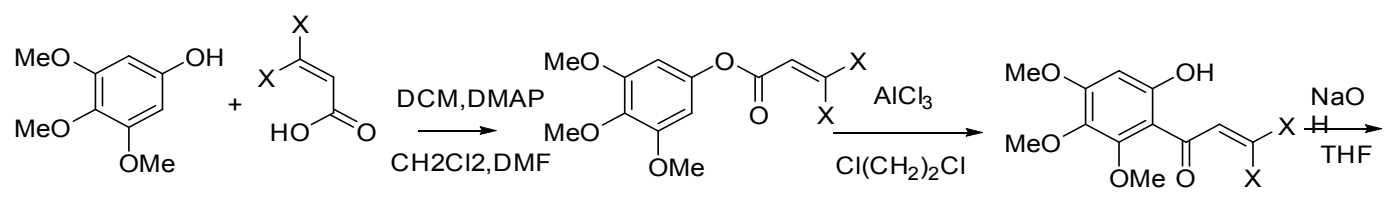

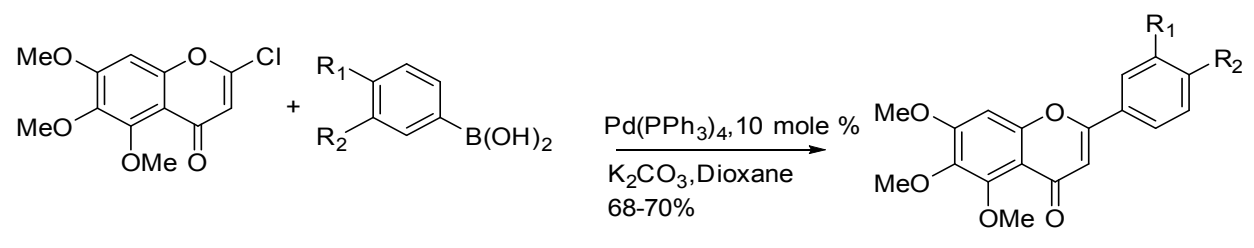

Scheme 36: Zambre\& Sangshetti used oxalic acid for oxidative coupling method ${ }^{44}$ 


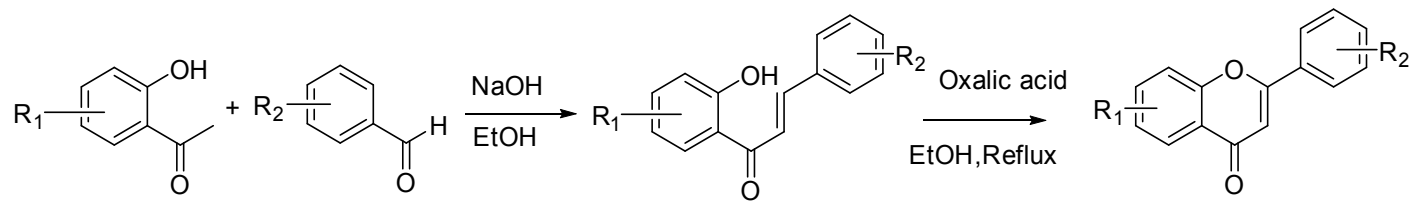

Scheme 37: lodine is used as catalyst for both Clause-Schmit condensation and oxidative coupling ${ }^{45}$

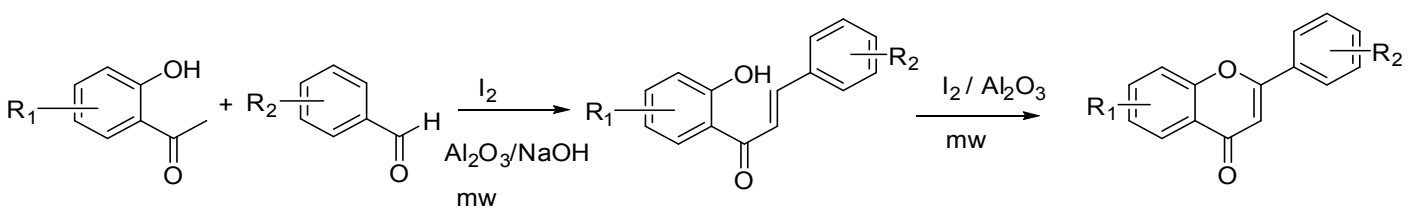

Scheme 38: Bosale \& Sarda used ionic liquid for the synthesis of flavones from dione intermediate ${ }^{46}$

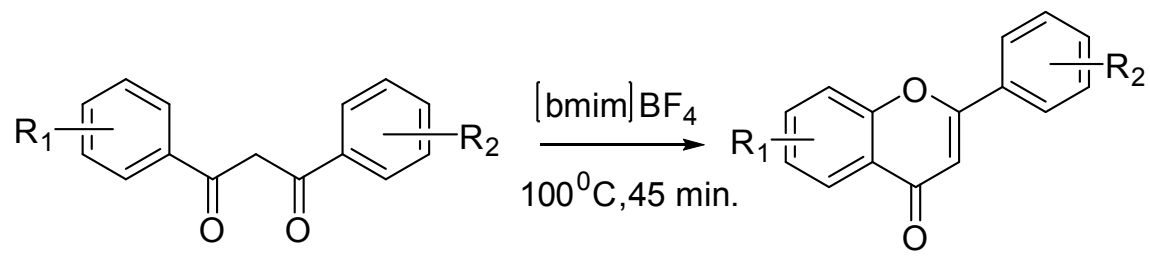

Scheme 39: Jae In Lee et $\mathrm{a}^{47}$ suggested the following methodology<smiles>[R]c1cc([R2])c(C(=O)O)c(O)c1</smiles><smiles>[R]c1ccc(C(=O)CC(=O)c2ccccc2)c([R])c1</smiles>

Scheme 40: Sodium tellurim oxide is used for the oxidativ coupling method by the author KumarS\& Sharma $D^{48}$<smiles>[R4]c1ccc(/C=C/C(=O)c2cc([R])c([R])cc2O)cc1[R]</smiles>

Scheme 41: New catalyst at present is use of hetro polyacid is used for the synthesis of flavones.This solvent free synthesis avoids excess loss of solvents ${ }^{49}$ 


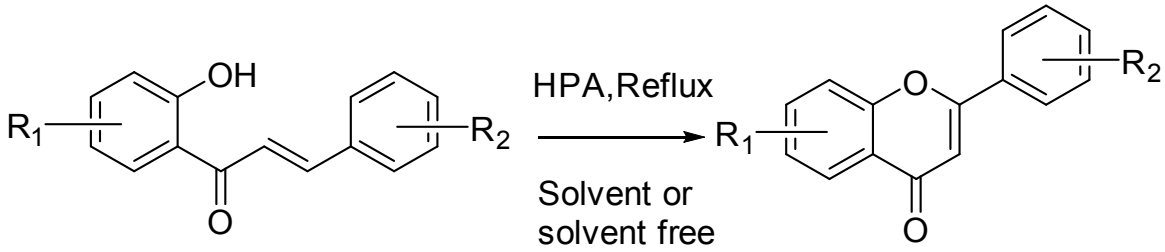

Scheme 42: Cul is another important catalyst invented by Zhiyun, Du \& Huifen N.. This method gives new catalyst for oxidative coupling of flavones ${ }^{50}$<smiles>[R]c1ccc(-c2cc(=O)c3ccc([R])cc3o2)cc1</smiles><smiles>[R]c1ccc(-c2ccc3c(=O)cc(-c4cccc([R])c4)oc3c2)cc1</smiles>

Scheme 43: Ortho acetyl acetophenone get converted to flavone directly without conversion to 1,3 dione intermediate ${ }^{51}$<smiles>CC(=O)c1ccccc1OC(F)F</smiles>

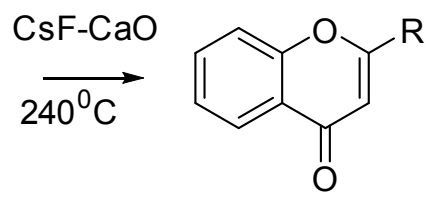

In conclusion I try to give most of the schems related to flavones. This review provides ready data for the people working in this field.

\section{REFERENCES}

1. Liu, H. L.; Jiang, W. B.; Xie, M. X. Recent Pat. Anti-Cancer Drug Discovery 5: 152-164 (2010).

2. Manthey, J. A.; Grohmann, K.; Montanari, A.; Ash, K.; Manthey, C. L. J. Nat Prod., 62: 441-444 (1999).

3. Maurya, R.; Rawat, P.; Sharan, K.; Siddiqui, J. A.; Swarnkar, G.; Mishra, G.; Manickavasagam, K.; Arya, R.; Chattopadhyay, N. World Pat. 110003 (2009).

4. Kunimasa, K.; Kuranuki, S.; Matsuura, N.;
Iwasaki, N.; Ikeda, M.; Ito, A.; Sashida, Y.; Mimaki, Y.; Yano, M.; Sato, M.; Igarashi, Y.; Oikawa, T. Bioorg Med. Chem. Lett. 19: 2062-2064 (2009).

5. Gabrielska, J.; SoczyDska-Kordala, M.; Przestalski, S. J. Agric. Food Chem. 53: 76-83 (2005).

6. Martens, S.; Mithofer, A. Phytochemistry, 66: 2399-2407 (2005).

7. Halliwell, B.; Aeshbach, R.; Loliger, J.; Aruoma, O. I.; Food Chem. Toxicol. 33: 601- 
617 (1995).

8. Ammar, N. M.; El-Diwany, A. I. J. Islamic Acad. Sci.1: $72-73$ (1988).

9. Hua M.; Zhen Y. Org. Lett., 2(12): 1765-1768 (2000).

10. Julio A. S.; Pilar V.T.; Raquel C.R., J. Org. Chem., 70(7): 2855-2858 (2005).

11. Emelia A. ; Alfredo C., Org. Lett., 11(15): 3210-3213 (2009).

12. Berta K.;Boris Š. Org. Lett., 9(20): 3993-3996 (2007)

13. Gustavo P.R.; Virla,E.G.; Duchowicz,P.R. Ana, L. G.; Diego M. R.; Daniel O. B., Erlinda del V. O. ; Autino, J.C. J. Agric. Food Chem., 58(10): 6290-6295 (2010).

14. Lydia, K.; Tomke, B.; Tobias, A.; Nigst, K.; Knochel,K J. Am. Chem. Soc., 134(33): 13584-13587 (2012).

15. Kumar,K.; Bodas M. S. Org. Lett., 2(24): 3821-3823 (2000).

16. Jie, Z.; Yufen,Z.; Hua, F. Org. Lett., 14 (11): 2710-2713 (2012).

17. Sashidhara,K.V. ;Kumar,M.; Kumar,A. Tetrahedron Letters, 53(18): 2355-2359 (2012).

18. Michael, L.; Kabir,M.S.; Cook,J.M. Tetrahedron Letters, 51(7): 1095-1098 (2010).

19. Kabalka,G.W. Mereddy,A.R. Tetrahedron Letters, 46(37): 6315-6317 (2005).

20. Das,J.; Ghosh,S. Tetrahedron Letters, 52(52): 7189-7194 (2011).

21. Bose,G.; Mondal,E.; Khan,A.T. ; Bordoloi,M. J. Tetrahedron Letters, 42(50): 8907-8909 (2001).

22. Lokhande,P.D.; Sakate,S.S.;Taksande, K. N.; Navghare,B. Tetrahedron Letters, 46(9): 15731574 (2005).

23. Kim,K.H.; Lee,H.S.; Kim,S.H. ;Kim,J.S. Tetrahedron Letters, 22(53): 2761-2764 (2012).

24. Jianming, L.; Muwen, L.;Yuanyuan ,Y.; Ningfei, Z.; Yuanli, Z.; Kelei, Z. Tetrahedron Letters, 54(14): 1802-1807 (2013).

25. Ganguly,A.K.; Kaur,S.; Mahata,P.K.; Biswas, D.; Pramanik,B.N. ; Chan,T.M. Tetrahedron Letters, 46(23): 4119-4121 (2005).

26. Maiti,M.; Karmakar,R.; Bhattacharya,R.N.; Kayal,K. Tetrahedron Letters, 52(43): 56105612 (2011).

27. Naseem Ahmed, Hasrat Ali, Johan E. van Lier
Tetrahedron Letters, 46(2):253-256 (2005.

28. Chin Fei Chee, Michael J.C. Buckle, Noorsaadah Abd. Rahman Tetrahedron Letters, 52(24): 3120-3123 (2011).

29. Mark Cushman, Dhanapalan Nagarathnam Tetrahedron Letters 31: 6497-6500 (1990).

30. V.N. Kalinin, M.V. Shostakovsky, A.B. Ponomaryov Tetrahedron Letters,31: 40734076 (1990).

31. Daniel ,D.; Laetitia, M. Tetrahedron Letters,36: 1845-1848 (1995).

32. Pinto,D.; Silva,A.S.; Cavaleiro,J.S. TetrahedronLetters, 35: 9459-9460 (1994).

33. Khan,A.T.; Goswami,P. Tetrahedron Letters, 46: 4937-4940 (2005).

34. Abu T. Khan, Abhik Choudhury, Shahzad Ali, Md. Musawwer Khan Tetrahedron Letters, 53: 4852-4857 (2012).

35. Yves Le, F.;Martine L. Tetrahedron Letters, 27: 5503-5504 (1986).

36. Frédéric Lassagne, Francis Pochat Tetrahedron Letters, 44(22): 9283-9285 (2003).

37. Dhanapalan N., Mark C. Tetrahedron, 47: 5071-5076 (1991).

38. Yoshida, Y.F.; Koya S.; Takayuki, D. Tetrahedron, 67(23): 9993-9997 (2011).

39. Francesco F.; Giosanna P.; Oriana P.; Ferdinando P. Tetrahedron 50(39): 1149911508 (1994).

40. Kumar,K.H.; Perumal,P.T. Tetrahedron, 63(38): 9531-9535 (2007).

41. Zanwar,M. R.; Raihan,M. J.; Gawande,S.D; Kavala,V; Janreddy, V.;Chun-Wei K.; Ambre,R. ; Ching-Fa,Y. J. Org. Chem., 77: 495-6504 (2012).

42. Eric F.; Dumas,A. M.; Kuropatwa, B. A.; Malhotra,N.R.; Sitler, T. C. J. Org. Chem., 71: 409-412 (2006).

43. Kraus, G.A.; Gupta, V. Org. Lett. 12: 52785280 (2010).

44. Zambare,A. S.; Sangshetti ,J. N. ; Kokare,N. D.; Shinde ,D. B. Chinese Chemical Letters 20: 171-174 (2009).

45. Sarda, S.R.;Jadhav,W. N. ; Pawar,R. P. International Journal of ChemTech Research 3: 539-543 (2009).

46. Bhosalea,R.S. ; Sardaa,S.R.; Girama, R.P.; Rauta,D.S; Parwea, S.P.; Ardhapurea, S.S.Pawarb,R.P. J.Iran.Chem.Soc., 6: 519512 (2009). 
47. Jae In Lee, Hwa Soo Son, Hyun Park Bull. Korean Chem. Soc. 25: 1945 (2004).

48. Kumar,S; Sharma ,D. Oriental Journal of Chemistry,27: 761-763 (2011).

49. Jahangir,G.M. ; Roshani, J.; Scheeren,W. 51. Bulgarian Chemical Communications 2(3):
210-216 (2010.

50. Zhiyun, Du; Huifen N; Kun, Zhang; Zengb,H; Wang, J. Org. Biomol. Chem., 9: 6930 (2011).

Didier, V.; Messaoud, H. React. Kinet. Catal. Lett., 72: 3-10 (2001). 